This item was submitted to Loughborough's Research Repository by the author.

Items in Figshare are protected by copyright, with all rights reserved, unless otherwise indicated.

\title{
SPH-BEM simulation of underwater explosion and bubble dynamics near rigid wall
}

PLEASE CITE THE PUBLISHED VERSION

https://doi.org/10.1007/s11431-018-9420-2

\section{PUBLISHER}

(c) Science China Press and Springer-Verlag

\section{VERSION}

AM (Accepted Manuscript)

\section{PUBLISHER STATEMENT}

This is a post-peer-review, pre-copyedit version of an article published in Science China Technological Sciences. The final authenticated version is available online at: https://doi.org/10.1007/s11431-018-9420-2.

\section{LICENCE}

CC BY-NC-ND 4.0

\section{REPOSITORY RECORD}

Zhang, Zhifan, Cheng Wang, A.M. Zhang, Vadim Silberschmidt, and Longkan Wang. 2019. "SPH-BEM Simulation of Underwater Explosion and Bubble Dynamics Near Rigid Wall". figshare. https://hdl.handle.net/2134/37177. 


\title{
SPH-BEM simulation of underwater explosion and bubble dynamics near rigid wall
}

\author{
ZHANG ZhiFan ${ }^{1 *}$, WANG Cheng ${ }^{1 *}$, ZHANG A-Man ${ }^{2}$, Silberschmidt Vadim $\mathrm{V}^{3}$ \& \\ WANG LongKan ${ }^{4}$ \\ ${ }^{1}$ State Key Laboratory of Explosion Science and Technology, Beijing Institute of Technology, Beijing 100081, China; \\ ${ }^{2}$ College of Shipbuilding Engineering, Harbin Engineering University, Harbin 150001, China; \\ ${ }^{3}$ Wolfson School of Mechanical, Electrical and Manufacturing Engineering, Loughborough University, Leicestershire LE11 $3 T U$, \\ UK; ${ }^{4}$ China Ship Research and Development Academy, Beijing 100101, China
}

\begin{abstract}
A process of underwater explosion of a charge near a rigid wall includes three main stages: charge detonation, bubble pulsation and jet formation. A smoothed particle hydrodynamics (SPH) method has natural advantages in solving problems with large deformations and is suitable for simulation of processes of charge detonation and jet formation. On the other hand, a boundary element method (BEM) is highly efficient for modelling of the bubble pulsation process. In this paper, a hybrid algorithm, fully utilizing advantages of both SPH and BEM, was applied to simulate the entire process of free and near-field underwater explosions. First, a numerical model of the free-field underwater explosion was developed, and the entire explosion processfrom the charge detonation to the jet formation-was analysed. Second, the obtained numerical results were compared with the original experimental data in order to verify the validity of the presented method. Third, a SPH model of underwater explosion for a column charge near a rigid wall was developed to simulate the detonation process. The results for propagation of a shock wave are in good accordance with the physical observations. After that, the SPH results were employed as initial conditions for the BEM to simulate the bubble pulsation. The obtained numerical results show that the bubble expanded at first and then shrunk due to a differences of pressure levels inside and outside it. Here, a good agreement between the numerical and experimental results for the shapes, the maximum radius and the movement of the bubble proved the effectiveness of the developed numerical model. Finally, the BEM results for a stage when an initial jet was formed were used as initial conditions for the SPH method to simulate the process of jet formation and its impact on the rigid wall. The numerical results agreed well with the experimental data, verifying the feasibility and suitability of the hybrid algorithm. Besides, the results show that, due to the effect of the Bjerknes force, a jet with a high speed was formed that may cause local damage to underwater structures.
\end{abstract}

\section{Introduction}

The process of underwater explosion [1-5] is a typical problem of transient fluid-structure interaction (FSI) in ship-

*Corresponding author (email: wangcheng@bit.edu.cn; heuzhangzhifan@hotmail. com) building and ocean engineering fields. Naval structures can be severely damaged by a near-field underwater explosion since a high-pressure shock wave and a pulsating bubble are generated during this process. Besides, due to the effect of the Bjerknes force, a high-speed jet can be induced near a structure, causing its local damage. Experiments were carried out by various researchers to investigate dynamics of 
bubbles generated by underwater explosions. Snay et al. $[6,7]$ experimentally investigated a migration of underwaterexplosion bubbles and its effect on the pulsation energy. Klaseboer et al. [8] carried out experiments to study expansion and collapse of a gas bubble as well as its damage of both rigid and resilient plates. Zhu et al. $[9,10]$ used highspeed photography to record an impulsion process of underwater-explosion bubbles and studied failure modes of a ship-type box structure subjected to a close underwater explosion. Chen et al. [11] conducted a series of comparative tests to study a dynamic response of a coated ship subjected to underwater explosion and analysed a protective effect of a rubber layer. Hung and Hwangfu [12] discussed dynamics of underwater-explosion bubbles near different boundaries based on a set of experiments, while Gong et al. [13] combined theoretical and experimental methods to investigate scaling relations for different types of bubbles. Li and Rong [14] studied load characteristics of an underwater explosion and a dynamic response of a cylindrical shell. Zhang et al. $[15,16]$ carried out a set of small-charge underwater explosion experiments to analyse the behavior of explosion bubbles under various boundary conditions. These experimental studies contributed to the investigation of development of underwater explosion. However, it was demonstrated that the underwater-explosion experiments had such shortcomings as safety challenges, low repeatability and limited information acquisition.

With the development of the computational capabilities, numerical methods were applied increasingly by researchers to investigate bubble dynamics in underwater explosions, employing such schemes as Runge-Kutta discontinuous Galerkin method [17], Arbitrary Lagrangian-Eulerian method [18], finite-volume method [19], and finite difference method [20]. The boundary element method (BEM) [21-24] is one of the most widely used approaches for solving problems of bubble dynamics and has well-known advantages in highly efficient simulations of a process of bubble pulsation. A bubble can transform from a singleconnected domain to a double-connected one as a jet penetrates the bubble. Hence, vortex sheet [25] or ring [26] models should be introduced into the bubble model to simulate dynamics of a final stage of bubble collapse. The vortex ring model is widely used to simulate the process of formation of toroidal bubbles. On the basis of this model, $\mathrm{Li}$ et al. $[27,28]$ applied the BEM to study bubble behavior near a free surface and a rigid body. Zhang et al. [29-32] investigated bubble dynamics near different boundaries with the BEM, and the obtained numerical results were compared with experimental data to verify the validity of the computational method. The BEM is effective to simulate bubble dynamics; however, additional numerical techniques should be used with the BEM model to simulate a process of jet formation. The SPH method with its meshless and La- grangian nature can overcome this limitation and is suitable for problems with large deformations. Hence, here SPH is used for simulations of the process of charge detonation and jet formation while the BEM is applied for the process of bubble pulsation. Thus, the entire process of the underwater explosion can be simulated with the suggested hybrid SPHBEM method.

In this paper, a hybrid algorithm [5] is developed based on a combination of the SPH method and the BEM to investigate pressure characteristics and bubble dynamics during the entire process of underwater explosion. First, a SPHBEM model of free-field underwater explosion is suggested. In order to verify the effectiveness of the presented algorithm, numerical results obtained for the shape and the maximum radius as well as the movement of a bubble are compared with experimental data. Based on this verification, a numerical model of a near-field underwater explosion for a column charge near a rigid wall is developed. The process of charge detonation is simulated with the SPH method and shock-wave propagation is analysed. After that, these $\mathrm{SPH}$ results are used as initial conditions for the BEM to simulate the process of bubble pulsation. The movement of the bubble is discussed and the numerical results are compared with the experimental ones to prove the validity of the suggested numerical method. Finally, the BEM results for formation of the initial jet are employed as initial conditions for the SPH method. Results for the jet formation, the impact process and the pressure characteristics at the rigid wall are analysed.

\section{SPH-BEM model}

\subsection{Basic SPH equations}

The SPH method (an established meshless scheme) [33-43] is suitable for solving problems with large deformations and can easily capture a medium interface. In this method, a calculation domain is discretized into a set of particles with material properties. A specially selected smoothing kernel function is utilized to transform Navier-Stokes equations into corresponding integral forms. The detailed solution for a standard SPH method can be found in ref. [33]. However, the standard SPH has poor accuracy at the interface between different media with a large density contrast [34]. Therefore, many SPH models [35-38] were proposed to solve problems with a large density contrast for simulations of multiphase flows. Hu and Adams [35] developed a new multi-phase projection formulation in order to get sharp discontinuities of density and viscosity. Grenier et al. [36] proposed an original variant of a gradient renormalization formula of the Shepard kernel to solve a problem of the discontinuity of the density at interfaces. Monaghan and Rafiee [37] slightly increased the pressure terms to stabilize the interface between different fluids. Chen et al. [38] offered a multiphase SPH model for 
multi-fluid flow with high density ratios. In this paper, based on a volume approximation, a detailed derivation of the modified SPH method [39] for the continuity, momentum and energy equations are given by

$\frac{\mathrm{d} \rho_{i}}{\mathrm{~d} t}=\rho_{i} \sum_{j=1}^{N} \frac{m_{j}}{\rho_{j}} \mathbf{v}_{i j}^{b} \cdot \frac{\partial W_{i j}}{\partial \mathbf{x}_{i}^{b}}$,

$\frac{\mathrm{d} v_{i}^{a}}{\mathrm{~d} t}=-\sum_{j=1}^{N} \frac{m_{j}}{\rho_{j}}\left(\frac{\sigma_{i}^{a b}+\sigma_{j}^{a b}}{\rho_{i}}+\Pi_{i j}\right) \frac{\partial W_{i j}}{\partial \mathbf{x}_{i}^{a}}$,

$\frac{\mathrm{d} e_{i}}{\mathrm{~d} t}=\frac{1}{2} \sum_{j=1}^{N} \frac{m_{j}}{\rho_{j}}\left(\frac{\sigma_{i}^{a b}+\sigma_{j}^{a b}}{\rho_{i}}+\Pi_{i j}\right) v_{i j}^{b} \frac{\partial W_{i j}}{\partial \mathbf{x}_{i}^{b}}$,

in which $\rho, t, m, \mathbf{v}, \mathbf{x}, \sigma$ and $e$ denote the density, time, mass, velocity, coordinates, stress and energy, respectively; $a$ and $b$ are the directions along the axes; $W_{i j}$ is the smoothing function of a pair of particles $i$ and $j$ (the cubic spline function is used in this paper); $\Pi_{i j}$ is the artificial viscosity.

Stress $\sigma$ for fluids can be decomposed into two terms: isotropic pressure and viscous shear stress. The isotropic pressure can be solved with an equation of state (EoS). The levels of temperature and pressure of explosive gas generated after explosive initiation are so high that the Jones-WilkinsLee (JWL) EoS [44] is used, given by

$p=A\left(1-\frac{\omega}{R_{1} v}\right) e^{-R_{1} v}+B\left(1-\frac{\omega}{R_{2} v}\right) e^{-R_{2} v}+\frac{\omega \rho_{0} e}{v}$,

in which $A, B, R_{1}, R_{2}$ and $\omega$ are the experimental fitting coefficients; $\rho_{0}$ and $e$ denote the initial density and detonation energy per unit mass; $v$ is the ratio of the initial density of the original explosive to the density of detonation products.

Due to high compressibility of water during the process of underwater explosion, a fit of Mie-Gruneisen EoS [45] for high-pressure conditions is used. If the water is in expansion state, i.e., $\mu<0$, the pressure can be obtained by

$p=\rho_{0} C_{0}{ }^{2} \mu+\left(\gamma_{0}+a \mu\right) e$,

in which $\rho_{0}, C_{0}, a, e$ and $\gamma_{0}$ denote the initial density, the sound velocity, the volume correction coefficient, the specific internal energy per unit volume and Gruneisen coefficient; $\mu$ is the compression ratio: $\mu=\eta-1$, where $\eta$ is the ratio of density before and after the explosion. As for the water in compressive state, i.e., $\mu>0$, the pressure is given by

$$
\begin{aligned}
p=\frac{\rho_{0} C_{0}^{2} \mu\left[1+\left(1-\frac{\gamma_{0}}{2}\right) \mu-\frac{a}{2} \mu^{2}\right]}{\left[1-\left(S_{1}-1\right) \mu-S_{2} \frac{\mu^{2}}{\mu+1}-S_{3} \frac{\mu^{3}}{(\mu+1)^{2}}\right]^{2}} \\
\quad+\left(\gamma_{0}+a \mu\right) e,
\end{aligned}
$$

in which $S_{1}, S_{2}$ and $S_{3}$ are the fitting coefficients. As for the viscous shear stress, the level of viscosity is so small that it can be ignored in analysis of underwater explosion with strong impacts.
A solid wall is one of the most common boundaries used in the SPH method in FSI problems, which should be dealt with since the numerical error can be caused by the boundary truncation. In this paper, a "dummy-particles" method proposed by Adami et al. [46] was applied for the boundary treatment. The solid boundary was composed of four layers of particles $w$. These boundary particles were searched in the calculation for the fluid particles $f$. The pressure and its normalization for the boundary particles $w$ can be drawn from [46]:

$$
\begin{aligned}
p_{w}= & p_{f}+\rho_{f}\left(\mathbf{g}-\mathbf{a}_{w}\right) \cdot \mathbf{r}_{w f}, \\
p_{w}= & \frac{\sum_{f} p_{f} W_{w f}+\left(\mathbf{g}-\mathbf{a}_{w}\right) \sum_{f} \rho_{f} \mathbf{r}_{w f} W_{w f}}{\sum_{f} W_{w f}},
\end{aligned}
$$

in which $p, \rho$ and a are the pressure, density and acceleration; $\mathbf{g}$ is the acceleration of gravity; $\mathbf{r}_{w f}$ is the displacement between the boundary and fluid particles; $W_{w f}$ is the smoothing function of a pair of particles $w$ and $f$.

The density $\rho_{w}$ for the solid boundary is obtained by [47]

$\rho_{w}=\rho_{r} \cdot\left(\frac{P_{w}}{B_{0}}+1\right)^{\frac{1}{\delta}}$,

in which the reference density $\rho_{r}$ is taken as $1000 \mathrm{~kg} / \mathrm{m}^{3} ; P_{w}$ is the initial water pressure; $B_{0}$ is the initial maximum pressure; the density ratio index $\delta$ is set to 7 .

\subsection{BEM method}

In the BEM, the fluid is assumed to be inviscid, irrotational and incompressible in simulations of an underwater explosion. The Laplace equation [48] is used, given by

$\nabla^{2} \Psi=0$

in which $\Psi$ is the velocity potential.

This velocity potential at any point in the fluid is expressed by that at the boundary and the normal derivative based on the Green equation. The boundary integration equation can be written as [48]

$\zeta \Psi(\mathrm{i})=\iint_{B}\left[\frac{\partial \Psi(\mathrm{j})}{\partial \mathbf{n}} \frac{1}{r_{i j}}-\Psi(j) \frac{\partial}{\partial n}\left(\frac{1}{r_{i j}}\right)\right] \mathrm{d} B$,

in which $\zeta$ is the solid angle; $B$ is the boundary of the fluid; $\mathbf{n}$ is the normal vector pointing out of the fluid domain; $i$ and $j$ are the field and source points at the boundary.

The kinematic and dynamic boundary conditions at the surface of the bubble are given as [48]

$\frac{\mathrm{d} \mathbf{R}}{\mathrm{d} t}=\nabla \Psi(x, y, z)$,

$\frac{\mathrm{d} \Psi}{\mathrm{d} t}=\frac{\nabla \Psi^{2}}{2}+\frac{P_{\infty}-P}{\rho}+g z$,

in which $P_{\infty}$ is the pressure far away from the charge centre 
in the horizontal direction; $\rho$ is the density of the fluid; $\mathbf{R}$ is the position vector at the boundary of the bubble; $P$ is the pressure inside the bubble.

The detailed description of the BEM can be found in ref. [48]. This highly efficient method was used to simulate the process of the bubble pulsation. Its results were subsequently taken as the initial conditions for the SPH calculation for the stage of jet formation; the linking procedure is discussed in the next section.

\subsection{Coupled SPH and BEM}

Variables such as coordinates, density and velocity are obtained for the particles in the SPH simulations. As for the BEM, magnitudes of these variables at the boundary of the bubble are used. In this paper, a hybrid algorithm [5] based on the SPH method and the BEM was adopted to investigate bubble dynamics near a rigid wall. The SPH method was first utilized to simulate the detonation process, and the obtained results were taken as the initial conditions for the BEM simulations of the bubble pulsation. After that, the BEM results were employed as the initial conditions for the SPH method to study the bubble motion during the process of jet formation. The detailed procedure is presented below.

\subsubsection{Bubble pulsation}

The main stages of the algorithm are as follows.

(1) The SPH results conversed to the BEM initial conditions

The hybrid SPH-BEM algorithm [5] was employed to simulate the entire process of the underwater explosion. The SPH models of free and near-field underwater explosions were developed to study the detonation process of the charge and the initial generation of the bubble. The "dummy-particles" method was used to treat the rigid boundary. The particle parameters including their coordinates, velocity, density and pressure at the boundary were obtained when the initial bubble was formed. Subsequently, the parameters at the interface were regarded as the initial conditions at the boundary for the BEM.

(2) The update for the BEM

Employing the above-mentioned initial conditions, a numerical model of the underwater-explosion bubble was developed to simulate the process of its pulsation. Parameters - pressure inside the bubble, the coordinates and the velocity at its boundary - were included into, and updated by, the BEM. The level of pressure inside the bubble was calculated based on the state equation of ideal gas. The coordinates at the boundary of the bubble were updated with the kinematic boundary conditions (eq. (12)). The velocity at its boundary was composed by normal and tangential components, obtained respectively by the boundary integration (eq. (13)) and differentiation of the velocity potential.

\subsubsection{Jet formation}

The BEM was applied in simulations of bubble dynamics during the stage of jet formation. The BEM results, once the initial jet was formed, were taken as the initial conditions for the SPH scheme.

The main stages of these algorithms were:

(1) Solutions for parameters inside the bubble

According to the conservation of mass, the initial density $\rho_{g}$ inside the bubble was given by

$\rho_{g}=\frac{W}{V_{g}}$

where $W$ and $V_{g}$ are the mass of the charge and the volume inside the bubble during the early stage of jet formation.

A linear interpolation was used in the surrounding water domain to get the initial velocity vector $\mathbf{v}_{g}$ inside the bubble. The initial pressure level calculated with the empirical equation $[49,50]$

$P_{g}=1.39 \times 10^{5} \cdot\left(\frac{W}{V_{g}}\right)^{\gamma}$,

with the specific heat capacity $\gamma$ set to 1.25 [29].

(2) Solutions for parameters in surrounding water

The values of parameters in the surrounding water domain were obtained with the BEM and the auxiliary function method [51] and used as the initial conditions for the SPH simulation, including the coordinates, velocity $\mathbf{v}_{w}$ and pressure $P_{w}$. The initial density of water $\rho_{w}$ was obtained with eq. (9).

\subsection{Numerical verification}

Based on the procedure described above, a numerical model of free-field underwater explosion was developed and the entire process, ranging from detonation to jet formation, was investigated. The obtained numerical results were compared with the experimental data in order to verify the validity of the hybrid algorithm.

\subsubsection{Experimental setup}

A small-scale underwater explosion experiment [16] was carried out to study the bubble behaviour for free-field underwater explosion during the process of its expansion, contraction and jet formation. Figures at different moments were captured in this experiment [16] to analyse the entire process of underwater explosion. The experimental equipment included a cubic water tank, high-speed cameras and data-acquisition systems. Here are the main features of the used setup.

(1) The tank was made of steel, with wall thickness of $10 \mathrm{~mm}$ and dimensions of $2 \mathrm{~m} \times 2 \mathrm{~m} \times 2 \mathrm{~m}$.

(2) A window made of PMMA (polymethyl methacrylate) was used to observe the experimental process. 
(3) The entire process of underwater explosion was recorded with high-speed cameras Phantom V12.1 and APXRS.

(4) A material of the column charge was PETN $\left(\mathrm{C}\left(\mathrm{CH}_{2}\right.\right.$ $\left.\left.\mathrm{ONO}_{2}\right)_{4}\right)$, with a mass of $4 \mathrm{~g}$; its diameter and length were $0.015 \mathrm{~m}$.

\subsubsection{Results and discussion}

In order to prove the effectiveness of the hybrid algorithm, a $\mathrm{SPH}$ model of the free-field underwater explosion was developed on the basis of the experimental setup (Figure 1). The particle sizes of the water and explosive domains were both $1.0 \mathrm{~mm}$. The particle numbers in these domains were 225 and 921375 , respectively. The entire process of the freefield underwater explosion was simulated, and shock-wave propagation and bubble dynamics were analysed.

The obtained experimental and numerical results for the pressure distribution are illustrated in Figure 2. The pressure distribution during the detonation process is shown in Figure 2(b-1) and (b-2). A shock wave was generated and propagated in the water, with the pressure first reaching $0.15 \mathrm{GPa}$ at about $0.134 \mathrm{~ms}$ and declining to about $0.09 \mathrm{GPa}$ at about $0.268 \mathrm{~ms}$. The explosion products expanded rapidly in the water, with the initial column shape developing into a nearspherical one. The pressure peak diminished sharply to about $0.1 \mathrm{MPa}$ at about $5.22 \mathrm{~ms}$ in Figure 2(b-3) with the expansion of the spherical bubble. The radius of the bubble reached its maximum at about $22.25 \mathrm{~ms}$ (Figure 2(b-4)); the experimental and numerical results for this value were $0.2705 \mathrm{~m}$ and $0.2633 \mathrm{~m}$, respectively, with an error lower than $3 \%$. The obtained computational results agreed well with the experimental data, proving the validity of the numerical method.

After that, the volume of the bubble began to decrease since pressure inside it was lower than that in the surrounding water. The pressure distributions at the upper and lower surfaces of the bubble no longer remained similar (Figure 2(b-5)). Besides, the lower surface of the bubble transformed from a spherical shape into a slightly flat one.
Under the effect of gravity, the lower surface of the bubble was gradually depressed towards the bubble's centre. Due to the pressure difference, an initial jet was formed in the direction opposite to that of gravity (Figure 2(b-6)). The jet nearly penetrated the bubble (Figure 2(b-7)). The pressure peak increased to about $23 \mathrm{MPa}$ with the decrease of the bubble's volume. This value was higher than that at the early stage of the jet formation as a result of the energy dispersion in the surrounding water. Subsequently, the bubble transformed into a double-connected domain (Figure 2(b-8)), resulting in formation of a toroidal bubble. The equivalent radius of the bubble reached the minimum. Then, pressure inside the bubble increased again and was higher than that in the surrounding water (Figure 2(b-9)), leading to secondary expansion of the bubble.

Consequently, the results obtained for the pressure distribution show that the main stages of the free-field underwater explosion-expansion, contraction and jet formation -were in accordance with the basic laws of bubble movement. The jet with a high speed was formed under the effect of gravity. Apparently, the numerical results for the bubble shape, the first cycle and the maximum radius of the bubble were in good agreement with the experimental data, confirming feasibility and effectiveness of the suggested hybrid algorithm.

Subsequently, the movement of the bubble was analysed; the respective evolution of displacements illustrated in Figure 3, with solid lines and markers corresponding to numerical and experimental results, respectively. The displacements at the top and the bottom of the bubble increased as it expanded to its maximum volume at first and then diminished. Its equivalent radius had a tendency similar to that of the displacement at the top. The bubble's centre moved slowly upwards during the expansion stage; this displacement increased significantly when the bubble volume reached the minimum. The error with the experiments was lower than $5 \%$, demonstrating a good agreement between the numerical and experimental results. It indicates
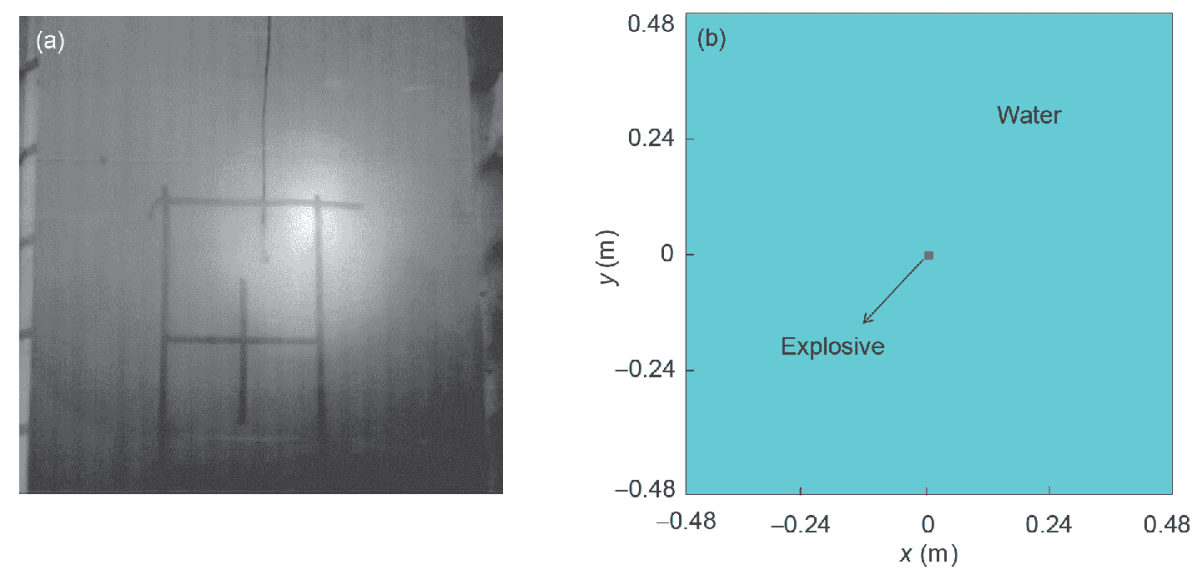

Figure 1 (Color online) Experimental (a) and numerical (b) models. 

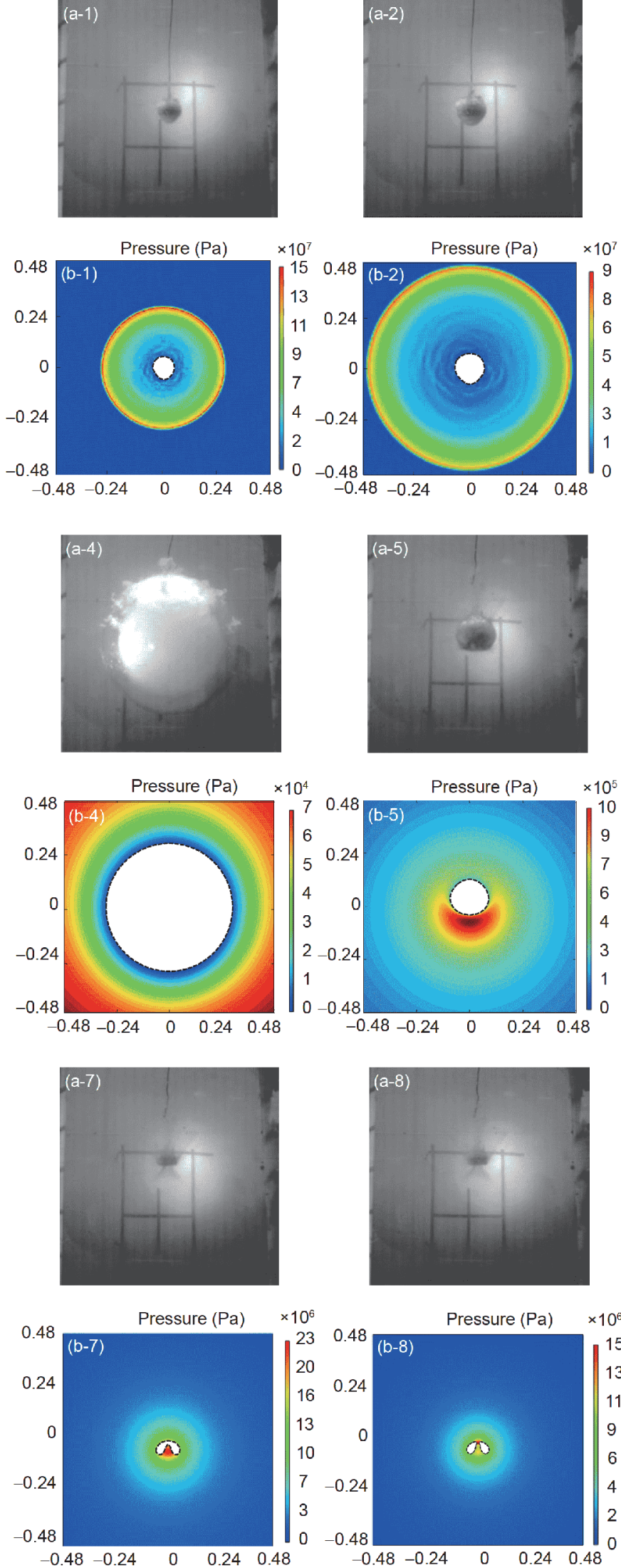
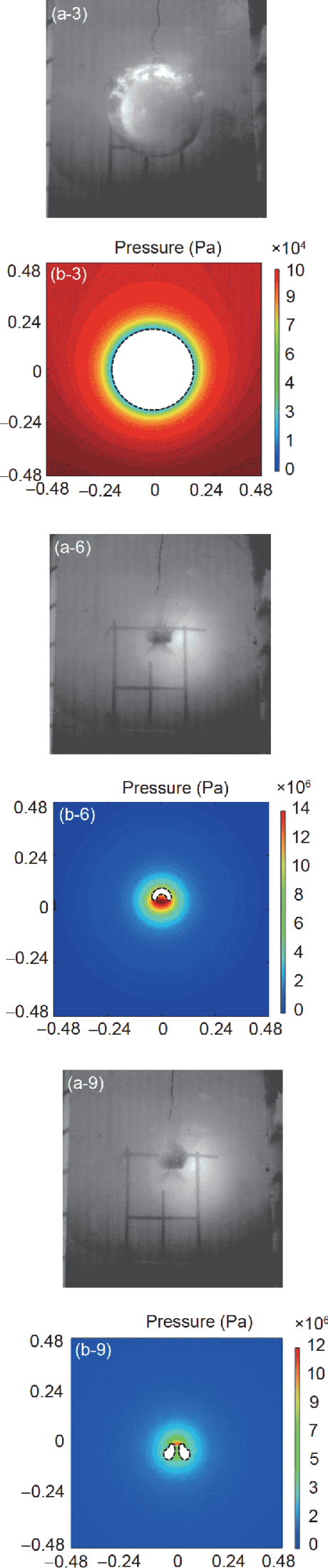

Figure 2 (Color online) Evolution of pressure distribution in entire process of free-field underwater explosion. (a-1)-(a-9) Experimental data corresponding to $0.13,0.27,5.20,22.04,43.23,43.66,43.86,44.04$ and $44.56 \mathrm{~ms}$; (b-1)-(b-9) numerical results corresponding to $0.13,0.27,5.22,22.25,43.43,43.70$, $43.91,44.23$ and $44.62 \mathrm{~ms}$. 


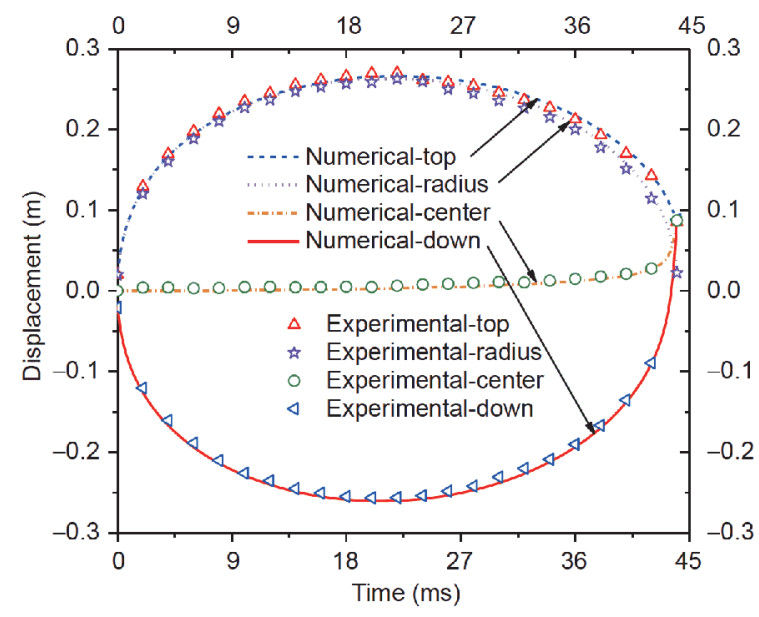

Figure 3 (Color online) Evolution of displacements at upper and lower surfaces of bubble and its centre.

that the presented hybrid algorithm was suitable for solving problems of underwater explosions.

\section{Dynamics of bubble near rigid wall}

After the validation of the developed hybrid approach, the effect of the rigid wall was studied. The bubble jet in the process of the free-field underwater explosion was caused by gravity, while for the near-field underwater explosion, due to the attractive force of the rigid wall, the process of jet formation was more complex. Additionally, the jet could cause secondary loading of structures. Hence, it is important to investigate the loading characteristics for the near-field underwater explosion - both shock-wave evolution and bubble dynamics. In this section, the hybrid scheme based on the combination of SPH and BEM was employed to simulate the propagation of shock waves and dynamics of bubbles near the rigid wall. The obtained numerical results are compared with the experimental data in order to verify the validity of the numerical method.

\subsection{Experimental setup and numerical model}

The analysed experiment was carried out by Zhang et al. [16]. A general setup for the test was the same as that in Sect. 2.4. Additionally, a rigid plate was fixed inside the box. The explosive was placed about $0.19 \mathrm{~m}$ above the plate. The numerical model reproduced the experimental setup and is discussed in the next section. The particle sizes of the water and the explosive domains were both $1.0 \mathrm{~mm}$. The particles numbers of the charge and the water were 225 and 642975 , respectively. The entire process of the near-field underwater explosion included three stages: detonation, bubble pulsation and jet formation. First, the SPH model of a column charge subjected to the near-field underwater explosion was devel- oped and propagation of the initial shock wave during the detonation process was analysed. Subsequently, the results obtained with the SPH method were used as the initial conditions for the BEM to simulate the process of bubble pulsation. Finally, the BEM results were used as the initial conditions for the SPH method in simulations of jet formation.

\subsection{Detonation process}

A comparison of the obtained experimental and numerical results for evolution of pressure distribution during the detonation process is given in Figure 4. The column charge was placed in the water tank at a distance of about $0.19 \mathrm{~m}$ above the plate (Figures 4(a-1) and (a-2)). A shock wave (SW1) was generated and propagated in the water after the charge was detonated. Subsequently, a reflected shock wave (RSW1) was produced when SW1 reached the rigid wall (Figures 4(b-1) and (b-2)), with the pressure up to $0.7 \mathrm{GPa}$. After RSW1 arrived at the bubble, not only a transmission shock wave was generated in the bubble, but also a reflected rarefaction wave in the water because impedance of the bubble was lower than that of the water (Figures 4(c-1) and (c-2)). The bubble shape became spherical. The numerical results show a good agreement with the experimental ones, proving validity of the SPH method in simulations of the detonation process.

\subsection{Process of bubble pulsation}

The SPH results obtained for $t \approx 0.267 \mathrm{~ms}$ were used as the initial conditions for the BEM to simulate the process of the bubble pulsation; the obtained pressure distributions are shown in Figure 5. Initially, the bubble expanded rapidly due to the pressure difference inside and outside it. Its shape remained spherical since gravity and a buoyant force had little effect on bubble dynamics in this period. Subsequently, the bubble got a slightly flat shape (Figures 5(a-1) and (a-2)) when its lower surface reached the rigid wall. With the expansion of the bubble, this surface was squeezed, while the upper one retained its spherical shape (Figures 5(b-1) and (b$2)$ ). The radius of the bubble increased to its maximum; the error between the numerical and experimental results for it was lower than $6 \%$. After that, the bubble began to shrink, since its internal pressure exceeded the outer one. The shrinking velocity at the top of the bubble was lower due to the effect of the buoyancy force while the lower surface of the bubble shrinked rapidly due to the effect of the Bjerknes force. As a result, the bubble got a conical shape (Figures 5 (c-1) and (c-2)). A high-speed jet formed gradually at the top of the bubble as a result of gravity and attraction of the rigid wall (Figures 5(d-1) and (d-2)), with pressure higher at the upper surface of the bubble than that in the surrounding 

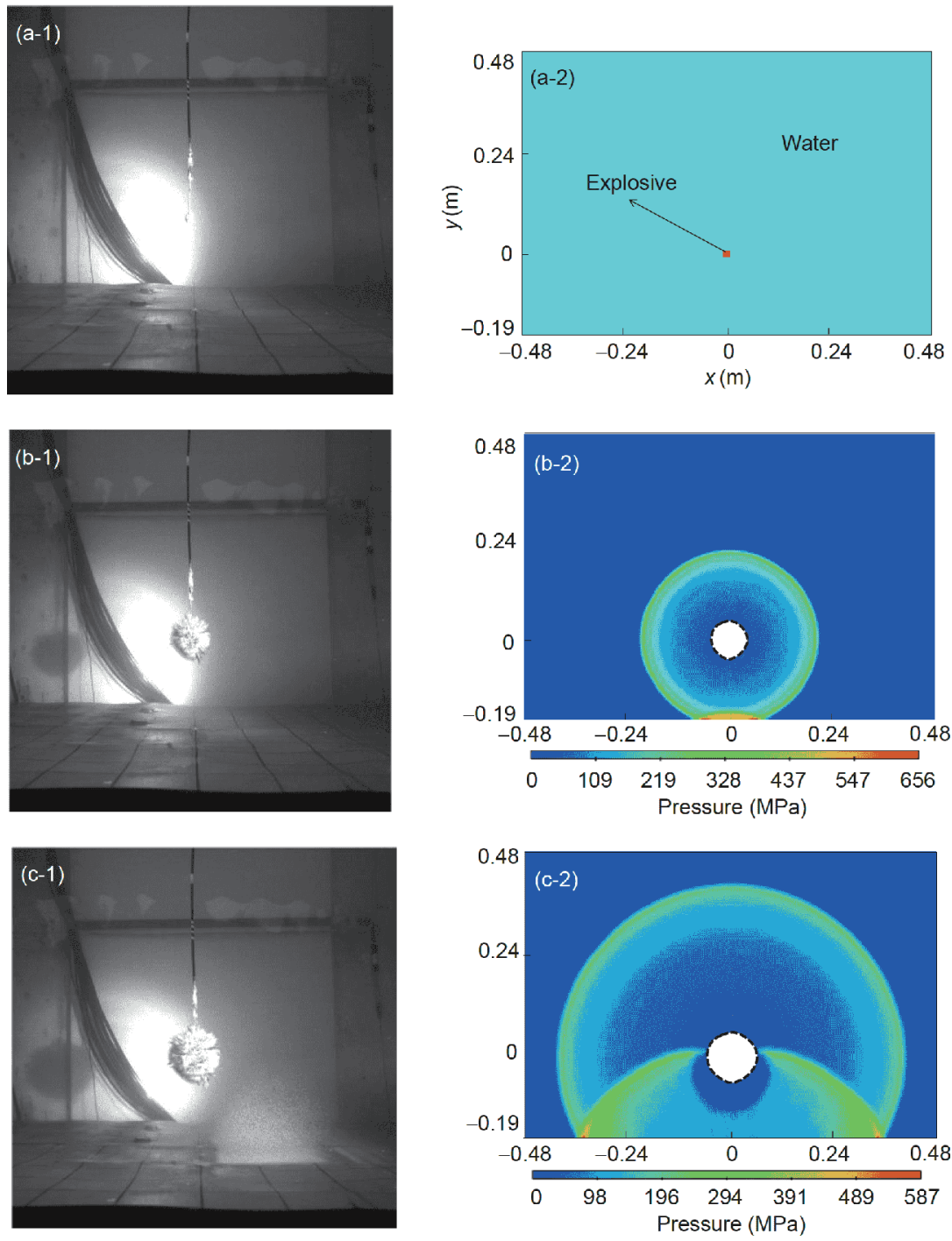

Figure 4 (Color online) Pressure distribution during process of charge detonation. (a-1)-(c-1) Experimental data [16] corresponding to $t \approx 0,0.133$ and $0.267 \mathrm{~ms}$; (a-2)-(c-2) numerical results corresponding to $t \approx 0,0.134$ and $0.268 \mathrm{~ms}$.

water. The numerical results agreed well with the experimental data, indicating that the BEM can solve effectively problems of bubble dynamics during the stage of bubble pulsation.

A character of bubble's movement (i.e., levels of displacements and velocity) at the surfaces of the formed bubble was studied after the analysis of its shape and pressure distributions in the fluid. The histories of displacements at the upper and lower surfaces of the bubble and its centre are presented in Figure 6. Apparently, the rigid wall had a great effect on displacements of the lower surface of the bubble. Its initial shape was spherical; after that, it expanded gradually since the level of pressure inside the bubble was higher than that in the surrounding water. The bubble subsequently flattened at its bottom after its lower surface reached the rigid wall. As for the centre of the bubble, it moved slightly in the direction opposite to the rigid wall during the expansion stage. After the volume of the bubble increased to its maximum, its centre tended to move in the direction of the rigid wall during the contraction stage. Once the jet was formed by the Bjerknes force, acting towards the wall, the bubble centre dropped rapidly. Additionally, it can be found that the experimental and numerical results for the maximum radius of the bubble were $0.2710 \mathrm{~m}$ and $0.2633 \mathrm{~m}$, respectively, i.e., with an error lower than $5 \%$. This good agreement of the results validates the BEM model.

Subsequently, the process of evolution of the velocities of the top and bottom bubble's points with time was analysed (Figure 7). Obviously, the velocities at these two points were in the opposite directions and increased rapidly at the early stage of bubble expansion, with a similar peak of about $114.7 \mathrm{~m} / \mathrm{s}$. Afterwards, the velocities gradually diminished. When the lower surface of the bubble reached the rigid wall, the velocity for the point at the bottom of the bubble van- 

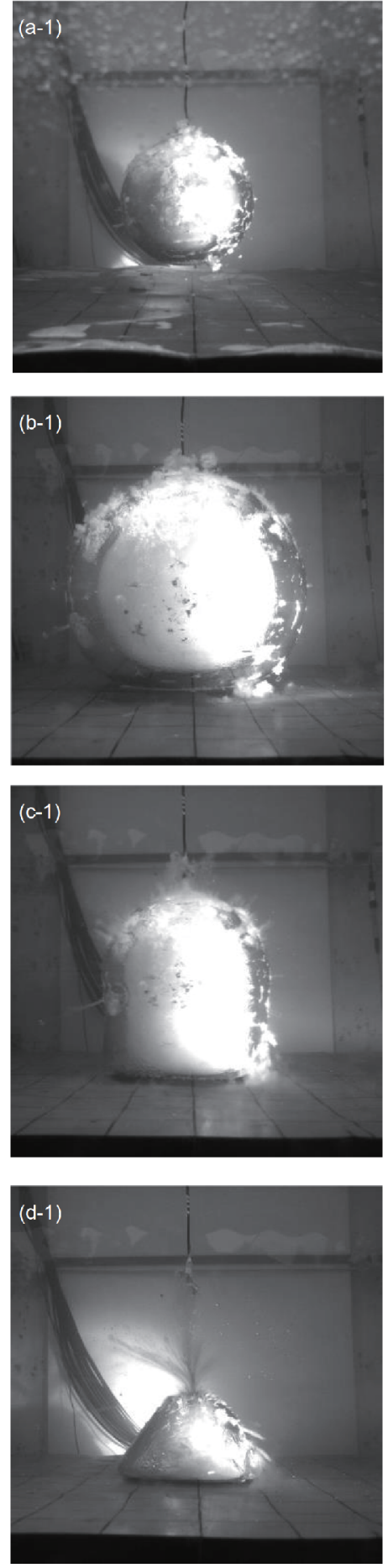
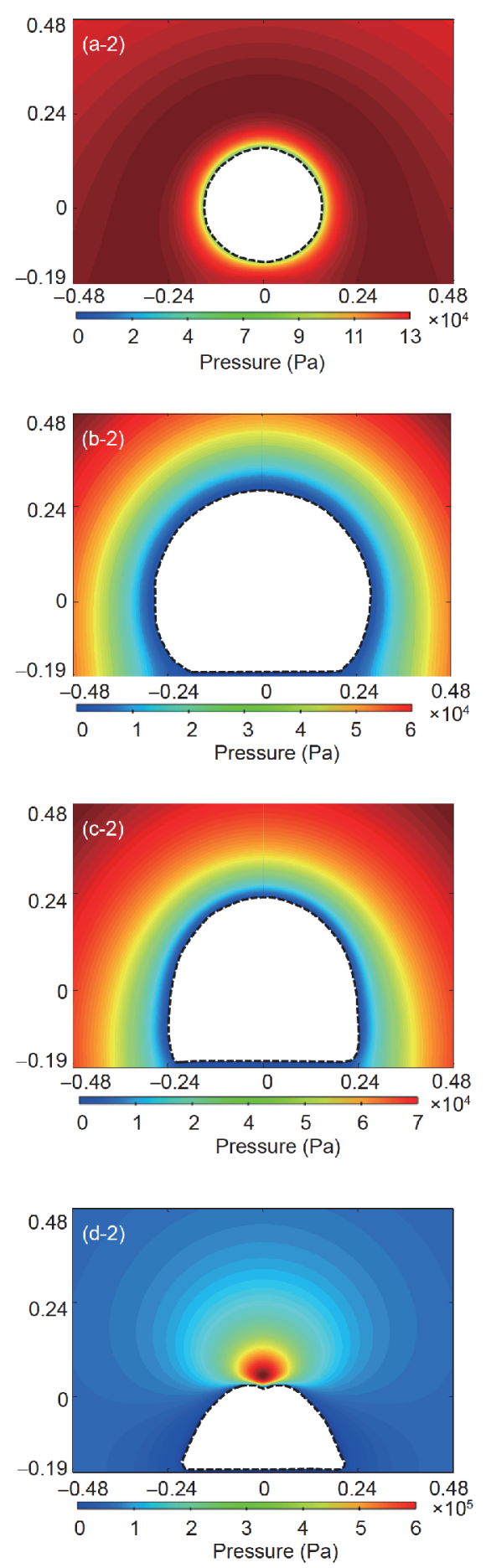

Figure 5 (Color online) Pressure distribution during process of bubble pulsation. (a-1)-(d-1) Experimental data [16] corresponding to $t \approx 3.33,22.53,37.73$ and $42.60 \mathrm{~ms}$; (a-2)-(d-2) numerical results corresponding to $t \approx 3.45,22.86,37.95$ and $42.84 \mathrm{~ms}$.

ished. As for the point at the top of the bubble, its velocity decreased to 0 when the radius of the bubble reached the maximum. After that, it began to increase in the opposite direction during the contraction stage of the bubble. The growth rate became higher at about $42.6 \mathrm{~ms}$ since the highspeed jet was formed; the velocity increased to about $90 \mathrm{~m} / \mathrm{s}$ at about $50 \mathrm{~ms}$.

\subsection{Process of jet formation}

A vortex ring model [26] should be introduced into the BEM since the bubble transformed from a single-connected domain to a double-connected one after the jet penetrated it. Hence, the SPH method was used to simulate the process of jet formation, using the obtained BEM results as the initial 


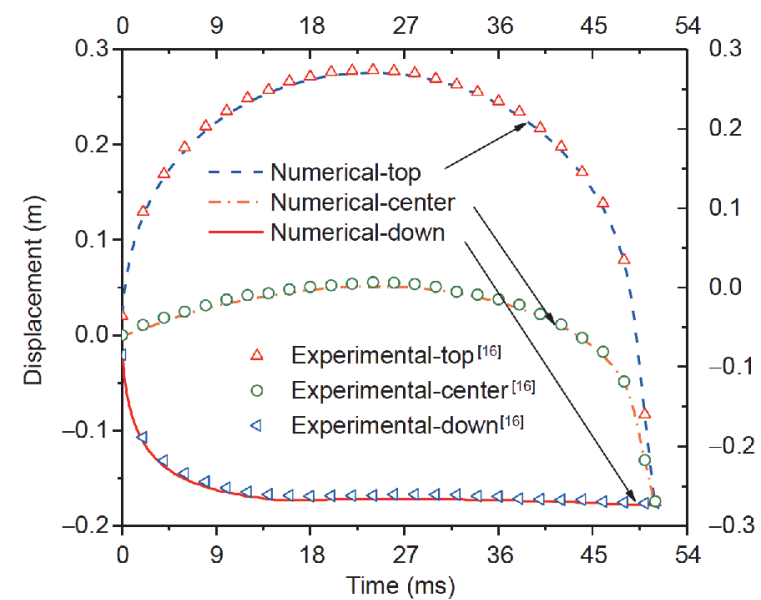

Figure 6 (Color online) Evolution of displacements at upper and lower surfaces of bubble and its centre.

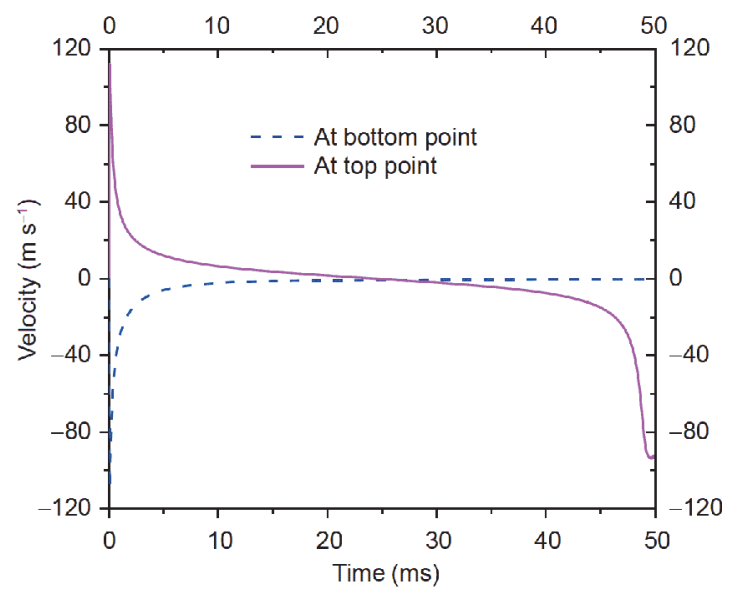

Figure 7 (Color online) Evolution of velocity at upper and lower surfaces of bubble.

conditions. The pressure distribution during the phase of the jet formation from the experiments and numerical simulations is shown in Figure 8.

A jet was formed in the direction towards the rigid wall under the effect of the Bjerknes force (Figures 8(a-1) and (a2)). After the jet arrived at the bottom of the bubble, the latter was gradually penetrated and developed its annular shape, changing from single-connected to double-connected. Subsequently, a high-pressure region was formed at the centre of the rigid wall due to the jet, with the pressure jumping to about $14 \mathrm{MPa}$ (Figures 8(b-1) and (b-2)); the respective experimental value at about $43.55 \mathrm{~ms}$ was about $13 \mathrm{MPa}$. The error between the numerical and experimental results was lower than $8 \%$, proving the effectiveness of the presented numerical model. After that, a compression wave caused by the jet formation arrived at the rigid wall. At the meantime, a rarefaction wave was generated in the direction of the inner jet, and the pressure declined (Figures $8(\mathrm{c}-1)$ and (c-2)), resulting in the formation of the wall-jet region. The respective numerical results were also in good agreement with the experimental data.

\section{Conclusions}

A strongly discontinuous shock wave, a non-spherical bubble and a high-speed jet can be generated in the process of near-field underwater explosion, which may cause severe damage to naval structures. These processes cannot be adequately described within the framework of a single numerical scheme, necessitating the development of a hybrid approach. Since the SPH method has advantages in solving problems with large deformations thanks to its meshless and Lagrangian nature and the BEM is highly efficient in simulations of the process of bubble pulsation, its combinations was employed. The hybrid algorithm based on the SPH method and the BEM was developed to simulate the entire process of the near-field underwater explosion, from detonation to jet formation. The obtained numerical results were compared with the original experimental data to verify the validity of the presented method. The following main conclusion can be drawn.

(1) A hybrid algorithm based on the combination of the SPH method and the BEM was used to simulate the entire process of the free-field underwater explosion. These two methods were employed to simulate the detonation process, the stage of jet formation and the pulsation process of the bubble. The numerical results were in good agreement with the experimental data, proving the feasibility and effectiveness of the hybrid modelling scheme.

(2) The bubble expanded due to higher pressure inside it than that in the surrounding fluid after the explosive was detonated in the free-field underwater explosion. After the radius of the bubble reached its maximum, the bubble began to shrink since the pressure inside it decreased. Under the effect of gravity, a high-speed jet was formed in the direction opposite to gravity.

(3) Based on simulations of the free-field underwater explosion, a numerical model of the near-field underwater explosion was developed to simulate propagation of the shock wave and dynamics of the bubble near a rigid wall. The SPH method was used first to simulate the detonation process. A reflected shock wave was generated after the direct shock wave arrived at the rigid wall. After this reflected wave reached the bubble, a transmission shock wave and a reflected rarefaction wave were respectively produced in the bubble and in the water. These results are in good accordance with the physics law of shock-wave propagation in a multilayer media.

(4) The BEM and the SPH method were respectively applied to simulate bubble pulsation and jet formation. The bubble shape developed from spherical to flat due to its 

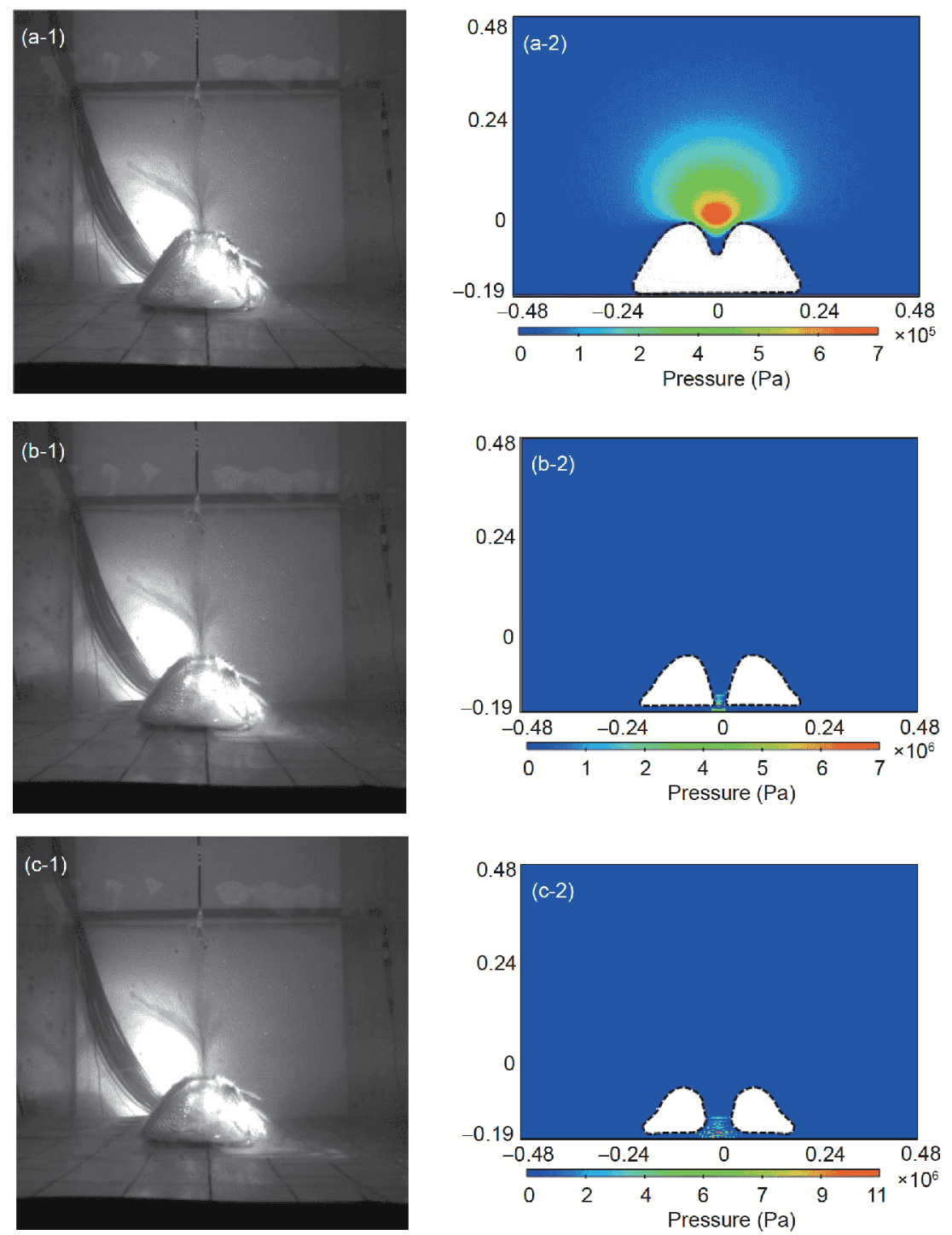

Figure 8 (Color online) Pressure distribution during process of jet formation. (a-1)-(c-1) Experimental data [16] corresponding to $t \approx 43.86,44.04$ and $44.56 \mathrm{~ms}$; (a-2)-(c-2) numerical results corresponding to $t \approx 43.91,44.23$ and $44.62 \mathrm{~ms}$.

compression at the rigid wall during the expansion stage. Subsequently, the high-speed jet was formed at the wall by the Bjerknes force at the stage of bubble contraction. A good agreement between the experimental and numerical results validated the hybrid algorithm.

(5) The developed hybrid algorithm, fully utilizing advantages of both SPH and BEM, is available to solve problems of the free and near-field underwater explosions, which can be used for other problems of fluid-structure interaction.

This work was supported by the National Natural Science Foundation of China (Grant Nos. 11802025 \& 11732003), China Postdoctoral Science Foundation (Grant No. 2017M620644), Science Challenge Project (Grant No. TZ2016001) and the State Key Laboratory of Explosion Science and Technology (Grant No. YBKT17-08). The authors would like to express their sincere thanks to Pu Cui for helpful experiments.
1 Hu J, Chen Z Y, Zhang X D, et al. Underwater explosion in centrifuge. Part I: Validation and calibration of scaling laws. Sci China Tech Sci, 2017, 60: 1638-1657

2 Yuan L, Zhou H Y, Liang X Q, et al. Underwater explosion in centrifuge. Part II: Dynamic responses of defensive steel plate. Sci China Tech Sci, 2017, 60: 1941-1957

3 Zhang Z, Wang L, Silberschmidt V V. Damage response of steel plate to underwater explosion: Effect of shaped charge liner. Int J Impact Eng, 2017, 103: 38-49

4 Cui $\mathrm{P}$, Zhang A M, Wang S P, et al. Ice breaking by a collapsing bubble. J Fluid Mech, 2018, 841: 287-309

5 Zhang A M, Yang W S, Huang C, et al. Numerical simulation of column charge underwater explosion based on SPH and BEM combination. Comput Fluids, 2013, 71: 169-178

6 Snay H G. Underwater Explosion Phenomena: The Parameters of Migrating Bubbles. NAVORD Report. 1962

7 Snay H G, Tipton R V. Charts for the Parameters of Migrating Explosion Bubbles. NAVORD Report. 1962

8 Klaseboer E, Hung K C, Wang C, et al. Experimental and numerical investigation of the dynamics of an underwater explosion bubble near 
a resilient/rigid structure. J Fluid Mech, 2005, 537: 387-413

9 Zhu X, Mu J L, Hong J B, et al. Experimental study of characters of bubble impulsion induced by underwater explosions. J Harbin Eng Univ, 2007, 28: 365-368

10 Wang $\mathrm{H}$, Zhu X, Cheng Y S, et al. Experimental and numerical investigation of ship structure subjected to close-in underwater shock wave and following gas bubble pulse. Mar Struct, 2014, 39: 90-117

11 Chen Y, Tong Z P, Hua H X, et al. Experimental investigation on the dynamic response of scaled ship model with rubber sandwich coatings subjected to underwater explosion. Int J Impact Eng, 2009, 36: 318 328

12 Hung C F, Hwangfu J J. Experimental study of the behaviour of minicharge underwater explosion bubbles near different boundaries. J Fluid Mech, 2010, 651: 55-80

13 Gong S W, Ohl S W, Klaseboer E, et al. Scaling law for bubbles induced by different external sources: Theoretical and experimental study. Phys Rev E, 2010, 81: 056317

14 Li J, Rong J L. Experimental and numerical investigation of the dynamic response of structures subjected to underwater explosion. Eur J Mech - B/Fluids, 2012, 32: 59-69

15 Zhang A M, Cui P, Cui J, et al. Experimental study on bubble dynamics subject to buoyancy. J Fluid Mech, 2015, 776: 137-160

16 Cui P, Zhang A M, Wang S P. Small-charge underwater explosion bubble experiments under various boundary conditions. Phys Fluids, 2016, 28: 117103

17 Park J. A coupled runge kutta discontinuous Galerkin-direct ghost fluid (RKDG-DGF) method to near-field early-time underwater explosion (UNDEX) simulations. Dissertation for the Doctoral Degree. Virginia Polytechnic Institute and State University, 2008. 1-130

18 Barras G, Souli M, Aquelet N, et al. Numerical simulation of underwater explosions using an ALE method. The pulsating bubble phenomena. Ocean Eng, 2012, 41: 53-66

19 Miller S T, Jasak H, Boger D A, et al. A pressure-based, compressible, two-phase flow finite volume method for underwater explosions. Comput Fluids, 2013, 87: 132-143

20 Hsiao C T, Jayaprakash A, Kapahi A, et al. Modelling of material pitting from cavitation bubble collapse. J Fluid Mech, 2014, 755: 142 175

21 Blake J R, Gibson D C. Growth and collapse of a vapour cavity near a free surface. J Fluid Mech, 1981, 111: 123-140

22 Wang Q X, Yeo K S, Khoo B C, et al. Strong interaction between a buoyancy bubble and a free surface. Theoret Comput Fluid Dyn, 1996, 8: $73-88$

23 Li S, Zhang A M, Wang S, et al. Transient interaction between a particle and an attached bubble with an application to cavitation in siltladen flow. Phys Fluids, 2018, 30: 082111

24 Klaseboer E, Khoo B C. Boundary integral equations as applied to an oscillating bubble near a fluid-fluid interface. Comput Mech, 2004, 33: $129-138$

25 Brujan E A, Keen G S, Vogel A. The final stage of the collapse of a cavitation bubble close to a rigid boundary. Phys Fluids, 2002, 14: 85103

26 Wang Q X, Yeo K S, Khoo B C, et al. Vortex ring modelling of toroidal bubbles. Theor Comput Fluid Dyn, 2005, 19: 303-317

27 Li Z R, Sun L, Zong Z, et al. A boundary element method for the simulation of non-spherical bubbles and their interactions near a free surface. Acta Mech Sin, 2012, 28: 51-65

28 Li Z, Sun L, Zong Z. Numerical analysis of gas bubbles in close proximity to a movable or deformable body. Arch Appl Mech, 2013, 83: $1715-1737$

29 Liu Y, Zhang A, Tian Z. Approximation of underwater explosion bubble by singularities based on BEM. Ocean Eng, 2014, 75: 46-52

30 Zhang A M, Liu Y L. Improved three-dimensional bubble dynamics model based on boundary element method. J Comput Phys, 2015, 294 : 208-223

31 Zhang A M, Wu W B, Liu Y L, et al. Nonlinear interaction between underwater explosion bubble and structure based on fully coupled model. Phys Fluids, 2017, 29: 082111

32 Liu N N, Cui P, Ren S F, et al. Study on the interactions between two identical oscillation bubbles and a free surface in a tank. Phys Fluids, 2017, 29: 052104

33 Liu G R, Liu M B. Smoothed Particle Hydrodynamics-A Meshfree Particle Method. Singapore: World Scientific Publishing Co. Pte. Ltd, 2003. $1-472$

34 Liu M B, Liu G R. Smoothed particle hydrodynamics (SPH): An overview and recent developments. Arch Computat Methods Eng, 2010, 17: 25-76

35 Hu X Y, Adams N A. An incompressible multi-phase SPH method. J Comput Phys, 2007, 227: 264-278

36 Grenier N, Antuono M, Colagrossi A, et al. An Hamiltonian interface SPH formulation for multi-fluid and free surface flows. J Comput Phys, 2009, 228: 8380-8393

37 Monaghan J J, Rafiee A. A simple SPH algorithm for multi-fluid flow with high density ratios. Int J Numer Meth Fluids, 2013, 71: 537-561

38 Chen Z, Zong Z, Liu M B, et al. An SPH model for multiphase flows with complex interfaces and large density differences. J Comput Phys, 2015, 283: 169-188

39 Zhang Z, Sun L, Yao X, et al. Smoothed particle hydrodynamics simulation of the submarine structure subjected to a contact underwater explosion. Combust Explos Shock Waves, 2015, 51: 502-510

40 Swegle J W, Attaway S W. On the feasibility of using smoothed particle hydrodynamics for underwater explosion calculations. Comput Mech, 1995, 17: 151-168

41 Zhang Z, Wang L, Ming F, et al. Application of smoothed particle hydrodynamics in analysis of shaped-charge jet penetration caused by underwater explosion. Ocean Eng, 2017, 145: 177-187

42 Cao X Y, Ming F R, Zhang A M, et al. Multi-phase SPH modeling of air effect on the dynamic flooding of a damaged cabin. Comput Fluids, 2018, 163: 7-19

43 Wang P, Zhang A M, Ming F, et al. A novel non-reflecting boundary condition for fluid dynamics solved by smoothed particle hydrodynamics. J Fluid Mech, 2019, 860: 81-114

44 Dobratz B M, Crawford P C. LLNL Explosive Handbook: Properties of Chemical Explosives and Explosives and Explosive Simulants. LLNL Report UCRL-52997. 198

45 Steinberg D J. Spherical Explosions and the Equation of State of Water. LLNL Technical Report UCID-20974. 1987

46 Adami S, Hu X Y, Adams N A. A generalized wall boundary condition for smoothed particle hydrodynamics. J Comput Phys, 2012, 231: 7057-7075

47 Monaghan J J. Simulating free surface flows with SPH. J Comput Phys, 1994, 110: 399-406

48 Zhang Y L, Yeo K S, Khoo B C, et al. 3D jet impact and toroidal bubbles. J Comput Phys, 2001, 166: 336-360

49 Cole R H. Underwater Explosion. New Jersey: Princeton University Press, 1948. 118-127

50 Wang C, Khoo B C. An indirect boundary element method for three-dimensional explosion bubbles. J Comput Phys, 2004, 194: 451480

$51 \mathrm{Wu}$ G X, Hu Z Z. Simulation of nonlinear interactions between waves and floating bodies through a finite-element-based numerical tank. Proc R Soc London Ser A-Math Phys Eng Sci, 2004, 460: 2797-2817 